\title{
Retracted: Electronic Clinical Decision Support Intervention to Increase Hepatitis C Screening and Linkage to Care Among Baby Boomers in Urban Safety Net Health Systems
}

\author{
Hilary Armstrong, MPH, ${ }^{1}$ Marisol Gonzalez-Drigo, RN, MPH, ${ }^{1}$ Gregory Norels, \\ Daniel Taussig, MA, William Trick, MD, ${ }^{2}$ Lisa Diep, MPH, ${ }^{2}$ Huiyuan Zhang, MS, ${ }^{2}$ \\ Jennifer Camacho Catrambone, MA, and Oluwatoyin Adeyemi, MD ${ }^{1}$
}

\begin{abstract}
The article entitled, "Electronic Clinical Decision Support Intervention to Increase Hepatitis C Screening and Linkage to Care Among Baby Boomers in Urban Safety Net Health Systems," by Armstrong et al., published online ahead of print (2019 Oct 8) in Population Health Management [doi: 10.1089/pop.2019 .0105], requires a retraction due to duplicate publication in the Journal of Community Medicine \& Health Education (JCMHE) in February of 2019, and then in Population Health Management in October of 2019. As it is against the standard protocols of peer review to publish original research in two different journals, Population Health Management is officially retracting the article from its literature. Population Health Management is dedicated to adhering to the policies and best practices of scientific publishing and the community it serves.
\end{abstract}

Keywords: Centers for Disease Control \& Prevention (CDC)/standards, hepatitis C virus, hepatitis C - chronic, mass screening, decision support techniques

\section{Introduction}

A N ESTIMATED 3.5 MiLlion PEOPLE in the United States are living with chronic hepatitis $\mathrm{C}$ infection $(\mathrm{HCV}) .{ }^{1-4}$ Approximately three quarters of persons chronically infected with HCV are baby boomers (born between 1945 and 1965), and the majority are unaware of their $\mathrm{HCV}$ status. ${ }^{1}$ As a result, the US Centers for Disease Control and Prevention (CDC) and US Preventive Services Task Force recommend universal 1-time HCV screening for baby boomers. ${ }^{1,5,6}$ Prior to these recommendations, most health systems used riskbased HCV screening to identify chronic infections, which often misses patients who do not self-report risk factors or who have not engaged in risk behaviors for many years. 5

Primary care providers have reported several barriers to implementing recommended screening practices, including time constraints, insufficient training on screening guidelines, and misconceptions about the CDC recommendations. ${ }^{7}$ To address these barriers, several studies have concluded that implementation of electronic clinical decision support (eCDS) tools is a low-cost and effective strategy for increasing age cohort screening in outpatient clinic networks. ${ }^{8-12}$ Additional clinical support available in primary care clinics, including disclosure of test results by a known and trusted provider, integrated support services, and systems for tracking patients from point of diagnosis to cure, indicate that this setting is optimal for HCV screening. ${ }^{10}$ Few studies have reported on patient outcomes from the point of screening to treatment completion.

\section{Methods}

Setting

Cook County Health $(\mathrm{CCH})$ consists of: 2 hospitals, a large network of more than a dozen community health centers, the Ruth M. Rothstein CORE Center, and Cermak Health Services, which provides health care to individuals in the Cook County correctional system. CCH serves

${ }^{1}$ Ruth M. Rothstein CORE Center, Cook County Health, Chicago, Illinois.

${ }^{2}$ Collaborative Research Unit, Cook County Health, Chicago, Illinois.

(c) Hilary Armstrong et al. 2019; Published by Mary Ann Liebert, Inc. This Open Access article is distributed under the terms of the Creative Commons License (http://creativecommons.org/licenses/by/4.0), which permits unrestricted use, distribution, and reproduction in any medium, provided the original work is properly cited. 
approximately 300,000 patients annually, the majority of whom are served at one of the outpatient clinics of the Ambulatory and Community Health Network (ACHN).

\section{Screening prompt}

Once Institutional Review Board approval was received for this quality improvement intervention, the study team created prompts for $\mathrm{HCV}$ testing using the CDS system embedded in the electronic medical record (EMR; Cerner Inc., Kansas City, MO). Prompt implementation required seeking and gaining approval from the chief medical officer and a standing Decision Support Committee. This initiative focused on primary care settings as a screening intervention for $\mathrm{HCV}$ by general practitioners to increase and routinize HCV screening among baby boomers, rather than on those completing treatment, such as gastroenterologists.

The rule was triggered for patients (1) born during 1945 through 1965, who (2) had never had an anti-HCV test, and who (3) had any order for a laboratory blood test. Final approval was obtained in February 2016 and the prompt was implemented in September 2016. In June 2017, the division chair of the microbiology and virology lab granted approval to implement $\mathrm{HCV}$ ribonucleic acid (RNA) testing.

\section{Data collection}

Data were abstracted from the EMR for 2 time periods: August 1, 2015 through August 31, 2016 (pre implementation of the eCDS tool) and September 1, 2016 through September 30, 2017 (post implementation of the eCDS tool). The eCDS tool was implemented across all $13 \mathrm{CCH}$ ACHN outpatient sites during September 2016. Data abstraction included age, sex, race, and ethnicity. Additional data abstraction for patients who received anti-HCV positive results included completion and result of HCV RNA confirmatory testing, completion of liver staging assessment, and treatment status (Figure 1). These data were abstracted from a separate patient database maintained by the Ruth M. Rothstein CORE Center Hepatitis C Clinic team. Linkage to care data for patients who received HCV staging and treatment at outside institutions are not included in this analysis

\section{Linkage to cart}

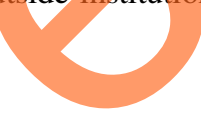

Patients with confirmed chronic HCV infection were referred to the Ruth M. Rothstein CORE Center for FibroScan (Echosens, Waltham, MA) staging and physician consult. Referrals submitted to the HIV/HCV care coordinator were reviewed to ensure all required laboratory tests were completed and then placed in a patient queue for scheduling. On average, patients received a FibroScan appointment within 3 weeks of referral. Once completed, FibroScan results were scanned into the EMR and/or faxed to external providers for ongoing $\mathrm{HCV}$ care. $\mathrm{CCH}$ patients were scheduled for initial assessments in the hepatitis clinic by an infectious disease provider. On average, patients received an appointment for an initial infectious disease physician assessment within 3 months.

Because of highly restrictive Medicaid reimbursement policies in Illinois, insurance coverage and fibrosis staging significantly determined which patients initiated the prior authorization process and started HCV treatment. As a result of these restrictions, 109 Stage 1 and Stage 2 patients were deemed ineligible for $\mathrm{HCV}$ treatment; of those patients $61.5 \%$ were male, $36.7 \%$ were female, and $1.8 \%$ were unknown. Most of the males were black/African Americans with an age range of $46-68(71.6 \%)$. Patients deemed ineligible for treatment because of low fibrosis staging, substance use, or unmanaged comorbidities were monitored and contacted periodically for reassessment.

Patients deemed eligible for HCV treatment met with a team that included a nurse coordinator, insurance specialist, a pharmacist, and prescribing providers to complete the prior authorization process, receive counseling on medication adherence and drug interactions, and schedule viral load monitoring appointments. Patients completed blood draws 4 weeks post initiation, at treatment completion, and 12 weeks post completion. Sustained virologic response data were not collected for patients who did not complete lab work 12 weeks post completion.

\section{Results}

\section{Screening}

The HCV eCDS prompt was implemented system-wide in September 2016. This study reports on 26 months of screening and linkage to care data from August 2015 to September 2017 (13 months pre implementation of eCDS and 13 month post implementation of eCDS). During the 26-month evaluation period, 15,630 patients were screened, with a greater proportion of women compared with men and blacks/African Americans representing the single largest racial group, followed by whites. Eighty percent of screened patients were born between 1944 and 1953, with the mean age of 60.5 years and median age of 60.0 years (Table 1).

The number of those newly tested each month increased from 2874 in the 13 months pre implementation to 12756 in the 13 months post implementation, representing a $344 \%$ increase. The percent of eligible patients tested increased by $24 \%$ across all sites. Some sites considered low-performing prior to implementation (ie, Site 3, Site 10) experienced significant increases in the percent of eligible patients screened $(38 \%$ and $33 \%$, respectively) while some high-performing sites (ie, Site 6) experienced more modest increases before reaching a screening plateau (20\% vs. $26 \%$ of eligible patients screened) (Figure 2). The number of patients previously screened (defined as the number of registered patients with a documented $\mathrm{HCV}$ test result in their health record, thereby excluding them from the prompt) increased by $22 \%$ across all ACHN sites.

As this metric only captures the number of patients who were excluded from the prompt among those with registered visits during the evaluation period, it does not accurately depict the number of patients previously tested for HCV. Markedly, the number of $\mathrm{HCV}$-antibody reactive patients identified increased from 265 patients in the pre-implementation period to 579 in the post-implementation period, representing a $118 \%$ increase across sites. A previously low-yield site (Site 2) experienced a $500 \%$ increase in $\mathrm{HCV}$-antibody reactive patients post implementation, highlighting the value and effectiveness of increased screening among age-based cohorts.

Implementation of HCV RNA reflex testing significantly increased the number of $\mathrm{HCV}$ antibody reactive patients who received confirmatory testing: $50 \%$ pre implementation vs. $100 \%$ post implementation. Additionally, reflex testing ultimately decreased wait times and increased patient 


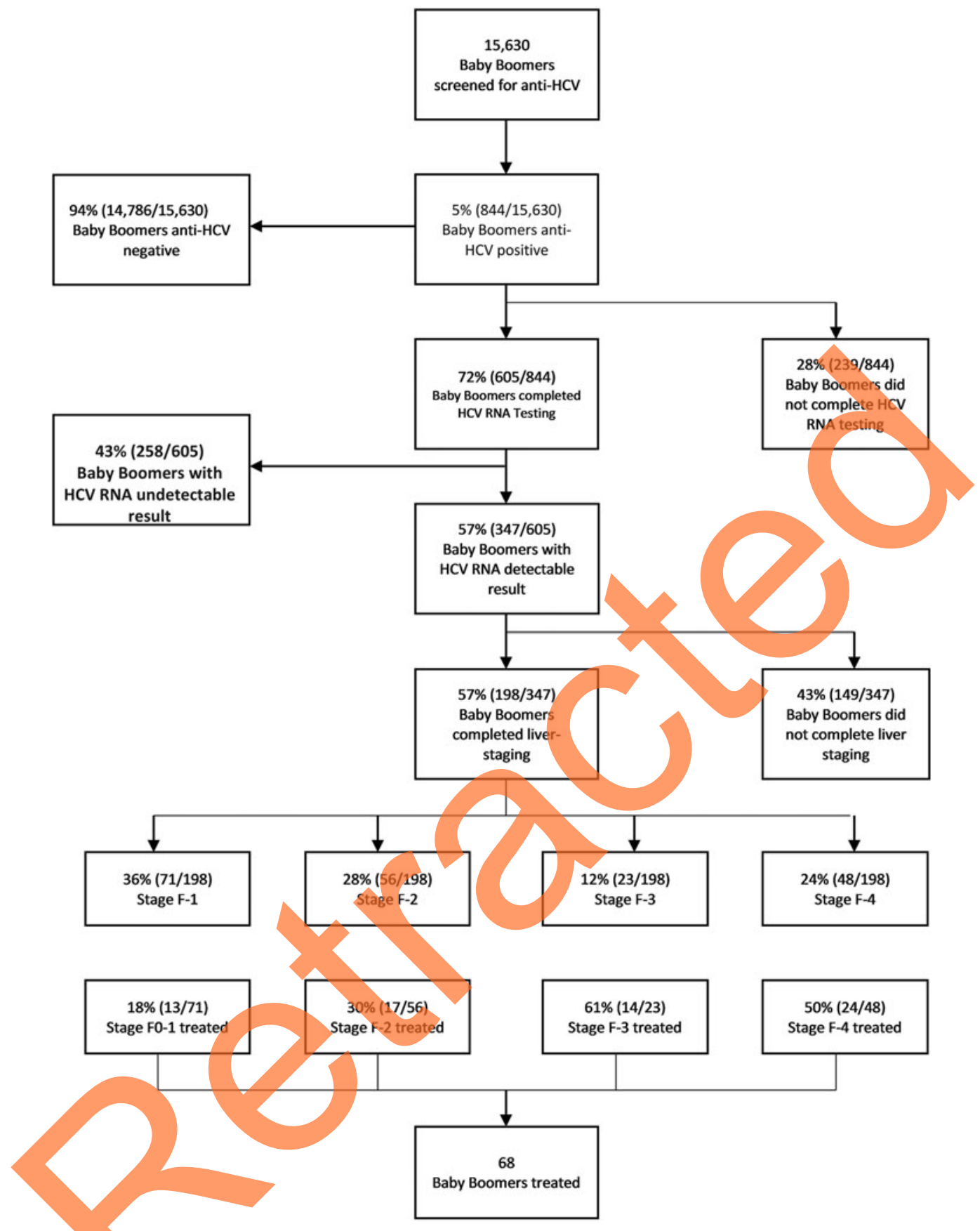

FIG. 1. Flow chart of baby boomers screened for anti-HCV through HCV treatment completion, Cook County Health, August 2015-September 2017. HCV, hepatitis C virus; RNA, ribonucleic acid.

capacity in hepatitis clinic appointments, as only patients with confirmed chronic $\mathrm{HCV}$ infection received referrals for physician consults.

\section{Linkage to care}

Between August 2015 and September 2017, 844 patients were identified as $\mathrm{HCV}$-antibody reactive. Patients identified as $\mathrm{HCV}$-antibody reactive were predominantly male, between the ages of 52 and 64, black/African American, and non-Hispanic (Table 1). Despite representing only $20 \%$ of patients screened, patients in the 52-64-year-old age group represented $70 \%$ of patients identified as HCV-antibody positive. Similarly, despite lower representation of males, black/African Americans, and non-Hispanics among the total screened population, males, black/African Americans and non-Hispanics disproportionately accounted for 59\%, $71 \%$, and $86 \%$ of $\mathrm{HCV}$-antibody reactive patients, respectively (Table 1).

There were no noticeable differences across age, sex, race, and ethnicity as to which patients completed HCV RNA confirmatory testing. Of those who completed HCV RNA testing, males were more likely than females to receive an HCV RNA detectable result $(64 \%$ vs $47 \%, P<.001)$ and blacks/African 
Table 1. Baby Boomer Hepatitis C Virus Care Continuum by Patient Characteristic, Cook County Health, August 2015-September 2017

\begin{tabular}{|c|c|c|c|c|c|c|}
\hline Patient characteristics & $\begin{array}{c}\text { Anti-HCV } \\
\text { screened (\%) }\end{array}$ & $\begin{array}{c}\text { Anti-HCV } \\
\text { positive (\%) }\end{array}$ & $\begin{array}{c}\text { Completed } \\
H C V R N A(\%)\end{array}$ & $\begin{array}{c}\text { HCV RNA } \\
\text { detected (\%) }\end{array}$ & $\underset{(\%)}{\text { Staged }}$ & $\begin{array}{c}\text { Treated } \\
(\%)\end{array}$ \\
\hline Total & 15,630 & 844 & 605 & 347 & 198 & 68 \\
\hline Mean age & 60.5 & 61.2 & 61.6 & 59.9 & 61.8 & 61.9 \\
\hline \multicolumn{7}{|l|}{ Age range } \\
\hline $52-64$ years & $3098(19.8)$ & $589(69.8)$ & $431(71.2)$ & $251(72.3)$ & $141(71.2)$ & $45(66.2)$ \\
\hline 65-74 years (Medicare eligible) & $12,532(80.2)$ & $255(30.2)$ & $174(28.8)$ & $96(27.7)$ & $57(28.8)$ & $23(33.8)$ \\
\hline \multicolumn{7}{|l|}{ Sex } \\
\hline Male & $6809(43.6)$ & $495(58.6)$ & $357(59.0)$ & $230(66.3)$ & $129(65.2)$ & $45(66.2)$ \\
\hline Female & $8819(56.4)$ & $349(41.4)$ & $248(41.0)$ & $117(33.7)$ & $69(34.8)$ & $23(33.8)$ \\
\hline \multicolumn{7}{|l|}{ Race } \\
\hline American Indian/Alaska Native & $315(2.0)$ & $4(0.5)$ & $3(0.5)$ & $2(0$. & $2(1.0)$ & $2(2.9)$ \\
\hline Asian & $796(5.1)$ & $23(2.7)$ & $14(2.3)$ & $6(1.7$ & $3(1.5)$ & $3(4.4)$ \\
\hline Black/African American & $8905(60.0)$ & $601(71.2)$ & $430(71.1)$ & $270(77$ & $150(75.8)$ & $49(72.1)$ \\
\hline Other/unknown & $1081(6.9)$ & $27(3.2)$ & $22(3.6)$ & $4(1.1$ & $2(1.0)$ & $1(1.5)$ \\
\hline White & $4532(29.0)$ & $189(22.4)$ & $136(22.5)$ & $65(18$ & $41(20.7)$ & $13(19.1)$ \\
\hline \multicolumn{7}{|l|}{ Ethnicity } \\
\hline Hispanic/Latino & $3955(25.3)$ & $115(13.6)$ & $84(13.9)$ & & $20(10.1)$ & $7(10.3)$ \\
\hline Not Hispanic/Latino & $11,675(74.7)$ & $729(86.4)$ & $521(86.1)$ & $318(9$ & $178(89.9)$ & $61(89.7)$ \\
\hline
\end{tabular}

Americans were more likely than whites to receive an HCV RNA detectable result $(63 \%$ vs $48 \%, P<.005)$. Across ethnicity groups, non-Hispanics were more likely than Hispanics to receive an HCV RNA detectable result (62\% vs $35 \%$, $P<.001)$. Males were more likely than females to complete a FibroScan assessment ( $26 \%$ vs $20 \%, P<.05)$.

Of the 198 patients who completed a FibroScan liver staging assessment, 71 (36\%) scored F-1, 56 (28\%) scored F-2, $23(12 \%)$ scored F-3, and $48(24 \%)$ scored F-4. American Indian/Alaska Native and Asians were more likely to receive a score of F-4, while black/African Americans and whites were more likely to receive a score of F-1 (Table 2). Because of restrictive Medicaid reimbursement policies, only
$34 \%$ of patients had received treatment ( $53 \%$ of those F3/F4 vs $23 \% \mathrm{~F}-2)$.

\section{Discussion}

Implementation of an HCV eCDS tool led to a marked increase in $\mathrm{HCV}$ testing among birth cohort patients across ambulatory sites, demonstrating the effectiveness of EMR interventions to promote adherence to guideline-based recommendations throughout a large urban health system.

Acceptance of the eCDS prompt likely was facilitated by (1) buy-in from system and medical leadership, (2) on-site provider and medical staff trainings by hepatitis staff, and

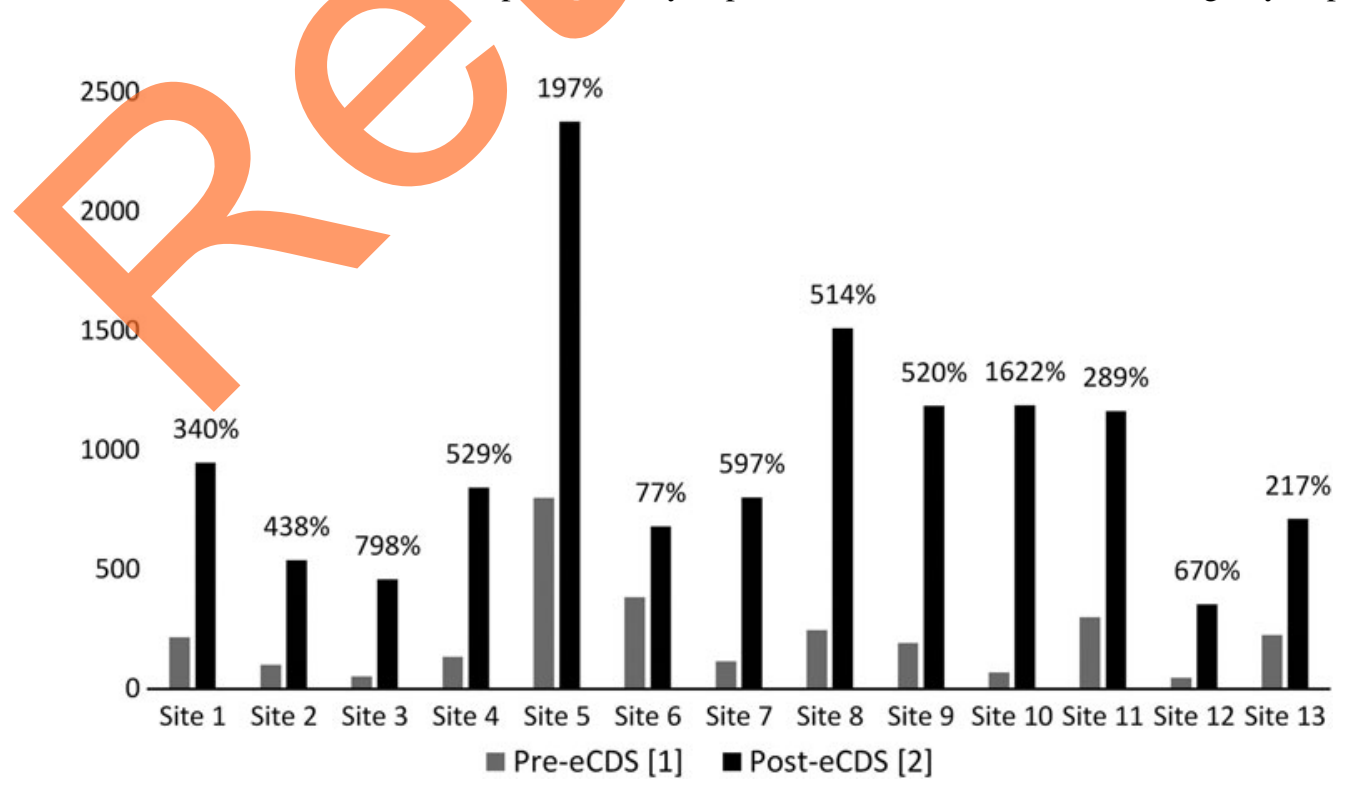

FIG. 2. Number of baby boomers screened for anti-HCV and percent increase, by site, pre and post implementation of eCDS, Cook County Health, August 2015-September 2017. [1] August 2015-August 2016. [2] September 2016-September 2017. eCDS, electronic clinical decision support; HCV, hepatitis C virus. 
(3) dissemination of site-specific data metrics to demonstrate progress toward goals and areas for improvement. Though provider adherence to the eCDS prompt immediately post implementation was promising, prompt fatigue and competing provider obligations may have led to a plateau effect approximately 8 months post implementation.

Notably, a lower than expected HCV RNA positivity (58\%) among HCV-antibody reactive patients was observed. Without extensive chart abstraction, it is difficult to determine whether this reflects spontaneous clearance trends or treatment completion at an external provider prior to $\mathrm{HCV}$ antibody screening at $\mathrm{CCH}$. Significantly, approximately one quarter of patients who completed fibrosis staging received a result of F4 , indicating that many had been living with $\mathrm{HCV}$ infection for several years. This highlights the urgency of universal screening for baby boomer patients as a tool to prevent disease progression and complications, including liver decompensation and hepatocellular carcinoma. According to the CDC, 1time testing of baby boomers may avert more than 120,000 HCV-related deaths. ${ }^{13}$

\section{Limitations}

This study had several limitations. The number of patients tested previously was derived from exclusionary criteria for the EMR prompt; monthly data grabs reported on the number of patients who met all other eligibility criteria for the prompt but had previously been tested. Because this metric does not distinguish between duplicated and unique patients, it should not be interpreted as an exact count of patients previously tested for HCV. Rather, this metric demonstrates that the number of patients excluded from the prompt because of previous testing has increased significantly since prompt implementation, indicating a good prompt saturation level throughout the patient population.

Additionally, data on the number of patients who completed stages of the care continuum beyond RNA confirmatory testing were only available for patients who continued care at $\mathrm{CCH}$. The study team was unable to capture linkage data on patients who received HCV care elsewhere. As a result, linkage to care and treatment outcomes likely were underreported.

Finally, these data were collected and analyzed before Illinois Medicaid removed all disease severity restrictions for $\mathrm{HCV}$ treatment coverage. Additional studies should be conducted to determine the impact on age, sex, and racial and ethnic disparities in access to HCV treatment after removal of these restrictions.

\section{Conclusions}

Implementation of eCDS tools across large urban safety net health systems is an effective strategy to ensure adherence to national guidelines for HCV screening among baby boomers. RNA reflex testing and timely access to liver staging assessments reduce the likelihood that patients are lost to care at subsequent stages of the care continuum. The large proportion of patients with advanced liver fibrosis identified through this intervention underscores the urgency of universal screening to prevent disease progression, improve patient outcomes, and reduce future health care costs.

As of November 2018, Illinois Medicaid has changed its rules to provide treatment for all $\mathrm{HCV}$ patients regardless of the stage of fibrosis-a public health service now available to all regardless of ability to pay. This demonstrates the importance of how state rules can have a positive impact on people's lives.

\section{Author Disclosure Statement}

The authors declare that there are no conflicts of interest.

\section{Funding Information}

This work was supported by grants from the following organization: The FOCUS Program is a public health initiative that enables partners to develop and share best practices in routine blood-borne virus (HIV, hepatitis C, hepatitis B) screening, diagnosis, and linkage to care in accordance with screening guidelines promulgated by the US Centers for Disease Control and Prevention, the US

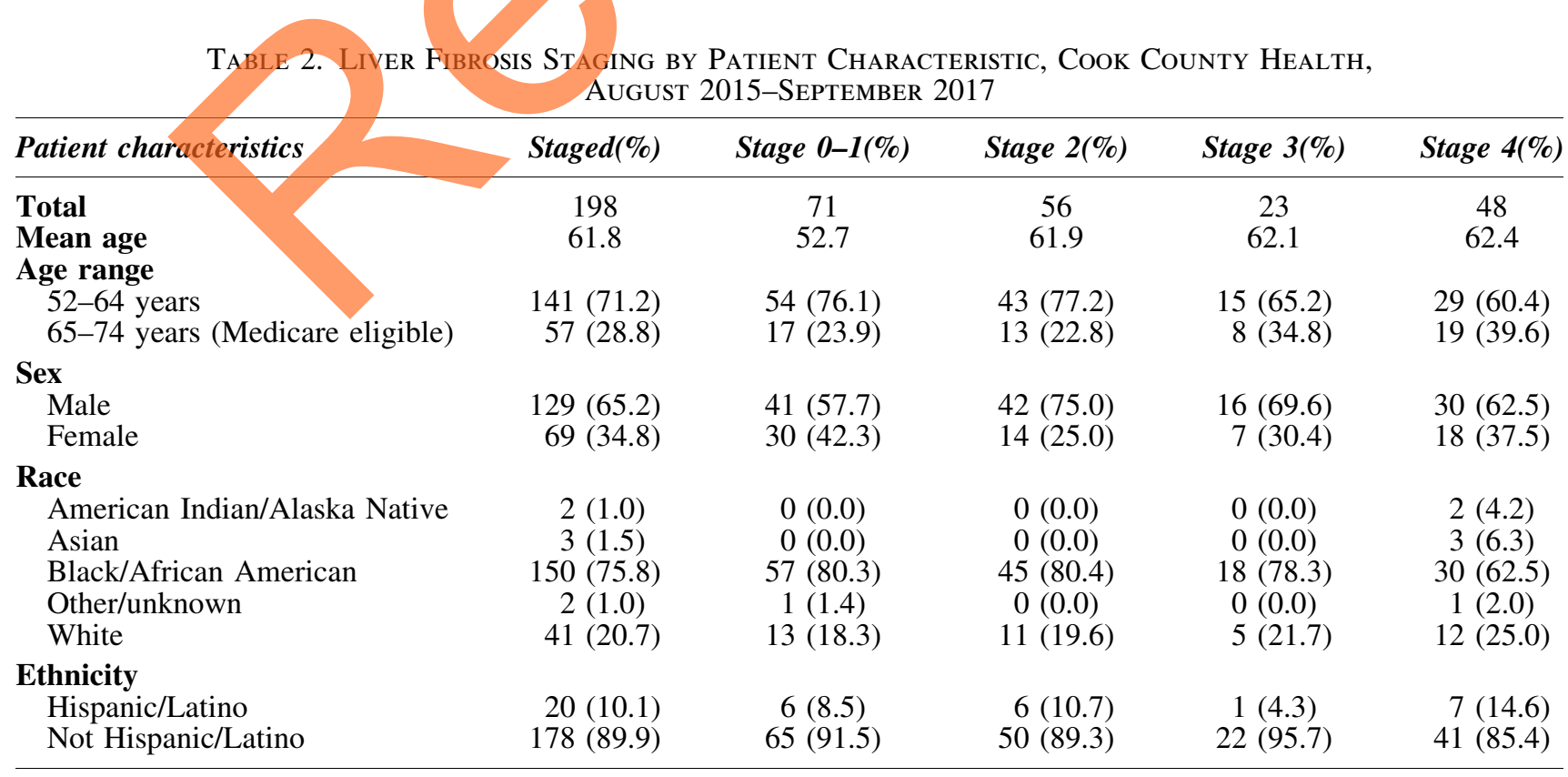


Preventive Services Task Force, and state and local public health departments. FOCUS funding supports HIV, HCV, and HBV screening and linkage to the first medical appointment after diagnosis; FOCUS funding does not support any activities beyond the first medical appointment and is agnostic to how FOCUS partners handle subsequent patient care and treatment.

\section{References}

1. Denniston MM, Jiles RB, Drobeniuc J, et al. Chronic hepatitis $\mathrm{C}$ virus infection in the United States, National Health and Nutrition Examination Survey 2003 to 2010. Ann Intern Med 2014;160:293-300.

2. Ward JW, Valdiserri RO, Koh HK. Hepatitis C virus prevention, care, and treatment: from policy to practice. Clin Infect Dis 2012;55 suppl 1:58-63.

3. Ward JW, Lok AS, Thomas DL, El-Serag HB, Kim WR. Report on a single-topic conference on "chronic viral hepatitis-strategies to improve effectiveness of screening and treatment." Hepatology 2012;55:307-315.

4. Denniston MM, Klevens RM, McQuillan GM, Jiles RB. Awareness of infection, knowledge of hepatitis $\mathrm{C}$, and medical follow-up among individuals testing positive for hepatitis C: National Health Nutrition Examination 20012008. Hepatology 2012;55:1652-1661.

5. Smith BD, Morgan RL, Beckett GA, et al. Recommendations for the identification of chronic hepatitis $\mathrm{C}$ virus infection among persons born during 1945-1965. MMWR Recomm Rep 2012;61(RR-4):1-32.

6. Moyer VA. Screening for hepatitis $C$ virus infection in adults: U.S. Preventive Services Task Force recommendation statement. Ann Intern Med 2013;159:349-357.

7. Jewett A, Garg A, Meyer K, et al. Hepatitis C virus testing perspectives among primary care physicians in four large primary care settings. Health Promot Pract 2015;16:256263.

8. Coyle C, Viner K, Hughes E, et al. Identification and linkage to care of $\mathrm{HCV}$-infected persons in five health centers-Philadelphia, Pennsylvania, 2012-2014. MMWR Morb Mortal Wkly Rep 2015;64:459-463.

9. Coyle C, Kwakwa H. Routine dual HCV and HIV testing: seroprevalence and linkage to care in four community health centers in Philadelphia, Pennsylvania. Public Health Rep 2016;131(suppl 1):41-52.

10. Coyle $\mathrm{C}$, Kwakwa $\mathrm{H}$, Viner $\mathrm{K}$. Integrating routine $\mathrm{HCV}$ testing in primary care: lessons learned from five federally qualified health centers in Philadelphia, Pennsylvania, 2012-2014. Public Health Rep 2016;131(suppl 2):65-73.

11. Sidlow $R$, Msaouel $P$. Improving hepatitis $C$ virus screening rates in primary care. J Healthc Qual 2015;37:319-323.

12. Miller LS, Rollin F, Fluker S, et al. High-yield birth-cohort hepatitis $\mathrm{C}$ virus screening and linkage to care among underserved African Americans, Atlanta, Georgia, 2012-2013. Public Health Rep 2016;131(suppl 2):84-90.

13. Division of Viral Hepatitis, National Center for HIV/AIDS, Viral Hepatitis, STD, and TB Prevention. People born 1945-1965 (Baby Boomers). https://www.cdc.gov/ hepatitis/populations/1945-1965.htm Accessed November 2,2018

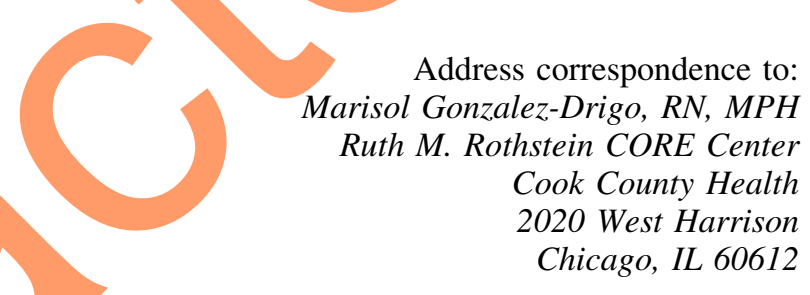

E-mail: mgonzalez@cookcountyhhs.org 